\title{
The aromatic fluctuation index (FLU): A new aromaticity index based on electron delocalization
}

\author{
Eduard Matito, Miquel Duran, and Miquel Solàa) \\ Institut de Química Computacional and Departament de Química, Universitat de Girona, 17071 Girona, \\ Catalonia, Spain
}

(Received 6 August 2004; accepted 5 October 2004; published online 14 December 2004)

\begin{abstract}
In this work, the aromatic fluctuation index (FLU) that describes the fluctuation of electronic charge between adjacent atoms in a given ring is introduced as a new aromaticity measure. This new electronic criterion of aromaticity is based on the fact that aromaticity is related to the cyclic delocalized circulation of $\pi$ electrons. It is defined not only considering the amount of electron sharing between contiguous atoms, which should be substantial in aromatic molecules, but also taking into account the similarity of electron sharing between adjacent atoms. For a series of rings in 15 planar polycyclic aromatic hydrocarbons, we have found that, in general, FLU is strongly correlated with other widely used indicators of local aromaticity, such as the harmonic-oscillator model of aromaticity, the nucleus independent chemical shift, and the para-delocalization index (PDI). In contrast to PDI, the FLU index can be applied to study the aromaticity of rings with any number of members and it can be used to analyze both the local and global aromatic character of rings and molecules. (C) 2005 American Institute of Physics. [DOI: 10.1063/1.1824895]
\end{abstract}

\section{INTRODUCTION}

There is no doubt about the fundamental role that aromaticity plays in current chemistry. ${ }^{1}$ Thus, the stability of certain molecular structures as well as many successes or failures of chemical reactions and changes in activation or reaction enthalpies are attributed to a gain or loss of aromaticity. Despite the lack of a clear and unambiguous definition of aromaticity, this chemical concept is deeply rooted in the chemistry community. ${ }^{1}$

Since aromaticity is not an observable and, therefore, not a directly measurable quantity, several definitions of aromaticity have been put forward. The most accepted definitions of aromaticity found in standard chemistry textbooks refer to the electronic cyclic delocalization present in all aromatic species. In this sense, Sondheimer considers aromatic molecules as those that have a "measurable degree of cyclic delocalization of a $\pi$-electron system." "Similarly, Schleyer and Jiao think of aromaticity as "associated with cyclic arrays of mobile electrons with favorable symmetries" while "the unfavorable symmetry properties of antiaromatic systems leads to localized rather than to delocalized electronic structures." ${ }^{3}$ This cyclic mobility of electrons is translated into characteristic aromatic manifestations such as bondlength equalization, abnormal chemical shifts, magnetic anisotropies, and energetic stabilization.

These particular manifestations of aromaticity have been taken into account to define different indices of aromaticity that evaluate the aromatic character of molecules based on their structural, magnetic, and energetic properties. ${ }^{4-9}$ The harmonic-oscillator model of aromaticity (HOMA) index,

\footnotetext{
a) Author to whom correspondence should be addressed.

Fax: (+34)-972-41-8356. Electronic mail: miquel.sola@udg.es
}

defined by Kruszewski and Krygowski ${ }^{10}$ and by Krygowski as $^{11}$

$$
\mathrm{HOMA}=1-\frac{\alpha}{n} \sum_{i=1}^{n}\left(R_{\mathrm{opt}}-R_{i}\right)^{2},
$$

is found to be among the most widespread and effective ${ }^{1,4}$ indices of aromaticity founded on structural criteria. In Eq. (1), $n$ is the number of bonds considered, and $\alpha$ is an empirical constant (for $\mathrm{C}-\mathrm{C}$ and $\mathrm{C}-\mathrm{N}$ bonds $\alpha=257.7$ and 93.52, respectively) chosen in such a way that $\mathrm{HOMA}=0$ for a model nonaromatic system, and HOMA $=1$ for a system with all bonds equal to an optimal value $R_{\text {opt }}(1.388$ and $1.334 \AA$ for $\mathrm{C}-\mathrm{C}$ and $\mathrm{C}-\mathrm{N}$ bonds, respectively) ${ }^{11}$ assumed to be achieved for fully aromatic systems. $R_{i}$ stands for a running bond length.

Magnetic indices of aromaticity are based on the $\pi$-electron ring current that is induced when the system is exposed to external magnetic fields. Probably the most widely used among this group is the nucleus independent chemical shift (NICS), proposed by Schleyer and co-workers. ${ }^{3,12}$ It is defined as the negative value of the absolute shielding computed at a ring center or at some other interesting point of the system. Rings with large negative NICS values are considered aromatic. The more negative the NICS values, the more aromatic the rings are.

Finally, indices of aromaticity based on energetic properties make use of the fact that conjugated cyclic $\pi$-electron compounds are more stable than their chain analogs. ${ }^{8,13}$ The most commonly used energetic measure of aromaticity is the aromatic stabilization energy, calculated as the reaction energy of a homodesmotic reaction. ${ }^{14}$

Less common is the use of measures of aromaticity founded on electronic properties. Among them, we can mention the highest-occupied-molecular-orbital-lowest-occu- 
pied-molecular-orbital gap, the absolute and relative hardness, the electrostatic potential, and the polarizability. ${ }^{5}$ In addition, descriptors of aromaticity using electronic parameters derived from the charge density have been also examined. Following this line of research, Jug defined a criterion based on bond orders ${ }^{15}$ and Howard and Krygowski ${ }^{16}$ proposed the density at the ring critical point interalia chargedensity descriptors.

More recently, several measures of delocalization derived from natural bond orbital analysis of the charge density have been used to quantify aromaticity in five-membered heteroaromatic compounds. ${ }^{17}$ In addition, the degree of $\pi$-electron delocalization in an aromatic compound obtained from an electron localization function ${ }^{18,19}$ (ELF) analysis has been proven to be a good aromaticity indicator, ${ }^{20}$ although no quantitative measures competing with existing indices have been given.

Lately, our group have employed electron delocalization measures derived from the second-order density ${ }^{21,22}$ as a new descriptor of local aromaticity. In particular, we have used the para-delocalization index (PDI), ${ }^{23}$ which is an average of all delocalization indices (DIs) of para-related carbon atoms in a given six-membered ring (6-MR). The DI value between atoms $A$ and $B, \delta(A, B)$, is obtained by the double integration of the exchange-correlation density over the basins of atoms $A$ and $B$ in the framework of the atoms in the molecules (AIM) theory of Bader (vide infra). ${ }^{24}$ Previous works ${ }^{23,25}$ have shown that for a series of planar and curved polycyclic aromatic hydrocarbons (PAHs), there is a satisfactory correlation between NICS, HOMA, and PDI. In general, larger PDIs go with larger absolute values of NICS and larger HOMA values.

The main inconvenience of PDI is that it cannot account for the aromaticity of rings having a number of members different from six. Moreover, it can only be used to analyze the local aromaticity of a given ring and not to describe the global aromatic character of a molecule having several fused rings. To surpass these shortcomings that limit the scope of the PDI measure, we have elaborated a new index also based on electron delocalization measures that can be applied to any ring or group of rings. In this sense, the new index defined here opens up entirely new possibilities for very detailed aromaticity studies.

Due to the multidimensional character of aromaticity, ${ }^{4,626,27}$ it is usually recommended to employ more than one aromaticity parameter for comparisons restricted to some regions or groups of relatively similar compounds. ${ }^{26}$ So far, the most widely used indices of aromaticity are based on structural, magnetic, and energetic measures. In this context, we believe that the definitions of this new aromaticity index and its $\pi$ counterpart derived from alternative indications of aromaticity, such as the electron delocalization, are very relevant.

\section{METHODOLOGY}

In this work we have used the negative of the exchangecorrelation density $\left[\Gamma_{\mathrm{XC}}\left(\vec{r}_{1}, \vec{r}_{2}\right)\right]$ as a function that accounts for electron sharing between two certain regions of the space, although, in principle, other functions that characterize elec- tron sharing could be used as well. Besides, we have employed the AIM theory ${ }^{24}$ to obtain an exhaustive physically sound subdivision of molecular space by means of a density topological analysis. Other partitions, such as the ELF division of space ${ }^{18,28}$ or the Mulliken-type partitioning in the Hilbert space spanned by the basis functions are also possible. Integration of the exchange-correlation (XC) density over two atomic basins ${ }^{24}$ yields atomic localization indices and DIs defined as ${ }^{21,22}$

$$
\lambda(A)=-\int_{A} d \vec{r}_{1} \int_{A} d \vec{r}_{2} \Gamma_{\mathrm{XC}}\left(\vec{r}_{1}, \vec{r}_{2}\right),
$$

and

$$
\delta(A, B)=-2 \int_{A} d \vec{r}_{1} \int_{B} d \vec{r}_{2} \Gamma_{\mathrm{XC}}\left(\vec{r}_{1}, \vec{r}_{2}\right),
$$

respectively.

The AIM topological analysis provides an exhaustive partition of molecular space, so these indices obey the following sum rule:

$$
\frac{1}{2} \sum_{A \neq B} \delta(A, B)+\lambda(A)=N(A),
$$

where $N(A)$ is the average population of an atom $A$ defined as follows:

$$
\langle N\rangle_{A}=N(A)=\int_{A} \rho(\vec{r}) d \vec{r} .
$$

The DI measure, $\delta(A, B)$, provides a quantitative idea of the number of electrons delocalized or shared between atoms $A$ and $B{ }^{22,29}$ It is worth noting that these indices are closely related to the fluctuation (or variance) in the average population of the basin of a given atom $A, \sigma^{2}$, defined by ${ }^{30}$

$$
\sigma^{2}[N(A)]=\left\langle N^{2}\right\rangle_{A}-\langle N\rangle_{A}^{2} .
$$

$\sigma^{2}[N(A)]$ represents the quantum-mechanical uncertainty on $N(A)$ and it is related to the localization and delocalization indices through the following equations: ${ }^{30,31}$

$$
\begin{aligned}
& \sigma^{2}[N(A)]=N(A)-\lambda(A), \\
& \sigma^{2}[N(A)]=\frac{1}{2} \sum_{B \neq A} \delta(A, B) .
\end{aligned}
$$

We can quantify the importance of the $A-B$ electron sharing/ fluctuation with respect to the total electron sharing/ fluctuation in atom $A$ using the following quantity:

$$
\operatorname{Flu}(A \rightarrow B)=\frac{\delta(A, B)}{\sum_{B \neq A} \delta(A, B)}=\frac{\delta(A, B)}{2[N(A)-\lambda(A)]} .
$$

If the molecule is aromatic, one can expect the transfer of electrons to be almost equal from $A$ to $B$ than from $B$ to $A$, so we can calculate the ratio of the electron exchange between $A$ and $B$ as

$$
\operatorname{Exc}(A-B)=\left[\frac{\operatorname{Flu}(A \rightarrow B)}{\operatorname{Flu}(B \rightarrow A)}\right]^{\delta} .
$$


The delta above is a simple function to make sure that the exchange term is always greater than or equal to 1 , so it takes the values

$$
\delta=\left\{\begin{array}{l}
1 \quad \operatorname{Flu}(A \rightarrow B)>\operatorname{Flu}(B \rightarrow A) \\
-1 \quad \operatorname{Flu}(A \rightarrow B) \leqslant \operatorname{Flu}(B \rightarrow A) .
\end{array}\right.
$$

This $\operatorname{Exc}(A-B)$ weighting factor gives an idea of the electron sharing similarity between $A$ and $B$ adjacent atoms. It will be close to 1 in aromatic species and particularly large in the case of molecules having atoms $A$ or $B$ with highly localized atomic charge. When multiplying the exchange factor above by the quantity of electrons shared by both atoms, we get a measure of $A-B$ net mobile charge. It is possible to find nonaromatic molecules having $\operatorname{Exc}(A-B)$ weighting factors close to 1 (for instance, cyclohexane) due to very similar or even equal electron sharing between adjacent atoms in the analyzed $\operatorname{ring}(\mathrm{s})$. For this reason, to correctly account for aromaticity, it is necessary to include in the expression a new factor $\left\{\left[\delta(A, B)-\delta_{\text {ref }}(A, B)\right] / \delta_{\text {ref }}(A, B)\right\}$ that compares the $A-B$ electron sharing with that found in a typical aromatic molecule, $\delta_{\text {ref }}(A, B)$. By squaring the product of these two factors for each connected pair of atoms in the ring and taking the average for all the pair of adjacent atoms in the ring, we obtain the following expression for the aromatic fluctuation index:

$\mathrm{FLU}=\frac{1}{n} \sum_{A-B}^{\text {ring }}\left\{\left[\frac{\operatorname{Flu}(A \rightarrow B)}{\operatorname{Flu}(B \rightarrow A)}\right]^{\delta}\left[\frac{\delta(A, B)-\delta_{\text {ref }}(A, B)}{\delta_{\text {ref }}(A, B)}\right]\right\}^{2}$,

which should give a number close to zero for any aromatic molecule. Abbreviated hereafter as FLU, this quantity not only analyzes the amount of electron sharing between adjacent atoms in a given ring, which should be substantial in aromatic molecules, but it also takes into account the similarity of electron sharing between adjacent atoms. In short, this index measures weighted electron delocalization divergences with respect to typical aromatic molecules. The FLU index as given by Eq. (12) requires the use of reference parameters. We anticipate here that for planar systems it is possible to define a FLU index that is free from this requisite (vide infra). It is worth repeating that although the FLU definition is unique, the quantity chosen to account for electron sharing or the topological approach to divide molecular space, i.e., molecular space portions assigned to each atom, may be different. Finally, it is also important to remark that, like the HOMA index, this dimensionless FLU quantity can be applied to analyze both local and global aromaticity.

\section{COMPUTATIONAL DETAILS}

Molecular geometries of systems analyzed have been fully optimized with the Hartree-Fock (HF) method using the $6-31 G(d)$ basis set $^{32}$ by means of the GAUSSIAN 98 program. ${ }^{33}$ The calculation of the electronic (FLU and PDI), geometric (HOMA), and magnetic (NICS) aromaticity criteria has been carried out at the same level of theory. To validate the level of accuracy of the results obtained with the 6-31G $(d)$ basis set, we have performed HF calculations for selected systems using the $6-311++G(d, p)$ basis set. ${ }^{34}$ The results, given as supporting information, show that increas- ing the quality of the basis set only produces minor changes that do not significantly affect the final conclusions.

The gauge-including-atomic-orbital method ${ }^{35}$ has been used to perform calculations of NICS. $\operatorname{NICS}(0)$ values have been computed at the ring centers determined by the nonweighted mean of the heavy atoms coordinates and at the ring critical point (RCP), ${ }^{24}$ the position of minimal charge density in the ring plane, as suggested by Cossio and co-workers. ${ }^{36}$ The results at the RCP reproduce those values calculated at the geometrical ring center, and for this reason, they are reported as supporting information. We have also computed NICS(1) values at $1 \AA$ above the molecular plane, where ring current contribution is larger and hence more representative of aromatic character. ${ }^{37}$ For those molecules with nonplanar structures we have fitted the best plane ${ }^{38}$ and have found the projection of the ring geometrical center to the fitted plane. Following the plane's normal vector direction to a $1-\AA$ distance from this site, we have reached the point to calculate the NICS(1) for nonplanar species. The NICS outof-plane component corresponding to the principal axis perpendicular to the ring plane, $\mathrm{NICS}_{z z}$, which has been recently recommended as a more direct measure of induced ring current densities, ${ }^{39}$ has been also calculated.

Integrations of DIs were performed by the use of the AIMPAC collection of programs ${ }^{40}$ through the following expression:

$$
\delta(A, B)=4 \sum_{i, j}^{N / 2} S_{i j}(A) S_{i j}(B) .
$$

The summations in Eq. (13) run over all the occupied molecular orbitals. $S_{i j}(A)$ is the overlap of the molecular orbitals $i$ and $j$ within the basin of atom $A$. The numerical accuracy of the AIM calculations has been assessed using two criteria: (i) the integration of the Laplacian of the electron density $\left[\nabla^{2} \rho(r)\right]$ within an atomic basin must be close to zero and (ii) the number of electrons in a molecule must be equal to the sum of all the electron populations of a molecule, and also equal to the sum of all the localization indices and half of the delocalization indices in the molecule [Eq. (4) $].{ }^{29}$ For all atomic calculations, integrated absolute values of $\nabla^{2} \rho(r)$ were always less than 0.001 a.u. For all molecules, errors in the calculated number of electrons were always less than 0.01 a.u.

All systems studied in the present work contain only $\mathrm{C}-\mathrm{C}$ and $\mathrm{C}-\mathrm{N}$ bonds in the ring structure. As a reference of aromatic electron sharing to compute the FLU index [Eq. (12)], we will take the value of $\delta_{\text {ref }}(\mathrm{C}, \mathrm{C})$ in benzene and $\delta_{\text {ref }}(\mathrm{C}, \mathrm{N})$ in pyridine. At the HF/6-31G(d) level of theory, these values are $1.4 e$ and $1.2 e$, respectively.

\section{RESULTS AND DISCUSSION}

This section is organized as follows. First, we analyze the performance of the FLU index for the assessment of local aromaticities in the series of PAHs depicted in Fig. 1. This figure contains also the labels given to each PAH and ring studied. The analysis will be carried out by comparing the FLU results with other widely used and different property- 
<smiles>c1ccccc1</smiles><smiles>c1ccc2ccccc2c1</smiles>

M1: Benzene M2: Naphthalene<smiles>c1ccc2cc3cc4ccccc4cc3cc2c1</smiles>

M4: Naphthacene

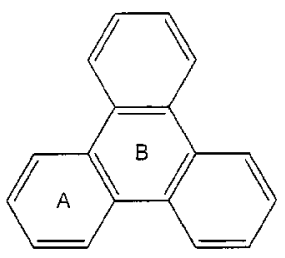

M6: Triphenylene

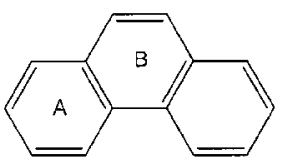

M8: Phenanthrene

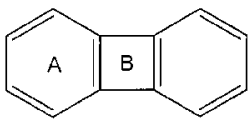

M10: Biphenylene<smiles>c1ccncc1</smiles>

M12: Pyridine<smiles>c1ccc2ncccc2c1</smiles>

M15: Quinoline<smiles>C1=CCCCC1</smiles><smiles>C1=CCC=CC1</smiles><smiles>C1CCCCC1</smiles>

M16: Cyclohexane

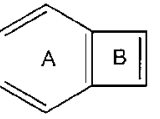

M11: Benzocyclobutadiene

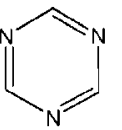

M14: Triazine

M13: Pyrimidine

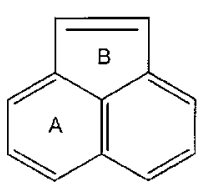

M9: Acenaphthylene

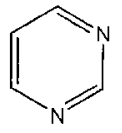

M14: Triazine 
TABLE I. HF/6-31G(d) calculated HOMA, NICS (ppm), PDI (electrons), and FLU values for the studied systems.

\begin{tabular}{|c|c|c|c|c|c|c|c|c|c|c|c|}
\hline & & EN & GEO & HOMA & $\operatorname{NICS}(0)$ & $\operatorname{NICS}_{z z}(0)$ & $\operatorname{NICS}(1)$ & $\operatorname{NICS}_{z z}(1)$ & PDI & FLU & $\mathrm{FLU}_{\pi}$ \\
\hline Benzene & M1 & -0.001 & 0.000 & 1.001 & -11.5 & -17.2 & -12.9 & -32.5 & 0.105 & 0.000 & 0.000 \\
\hline Naphthalene & M2 & 0.022 & 0.199 & 0.779 & -10.9 & -13.8 & -12.6 & -30.6 & 0.073 & 0.012 & 0.114 \\
\hline \multirow[t]{2}{*}{ Anthracene } & M3-A & 0.067 & 0.416 & 0.517 & -8.7 & -6.9 & -10.7 & -24.8 & 0.059 & 0.024 & 0.251 \\
\hline & M3-B & 0.045 & 0.072 & 0.884 & -14.2 & -21.9 & -15.5 & -38.4 & 0.070 & 0.007 & 0.024 \\
\hline \multirow[t]{2}{*}{ Naphthacene } & M4-A & 0.110 & 0.565 & 0.325 & -6.7 & -0.9 & -9.0 & -19.6 & 0.051 & 0.031 & 0.350 \\
\hline & M4-B & 0.073 & 0.153 & 0.774 & -13.8 & -20.4 & -15.2 & -37.2 & 0.063 & 0.011 & 0.071 \\
\hline \multirow[t]{2}{*}{ Chrysene } & M5-A & 0.011 & 0.130 & 0.859 & -11.1 & -13.7 & -12.8 & -30.7 & 0.079 & 0.008 & 0.067 \\
\hline & M5-B & 0.122 & 0.325 & 0.553 & -8.2 & -3.6 & -10.6 & -23.3 & 0.052 & 0.019 & 0.182 \\
\hline \multirow[t]{2}{*}{ Triphenylene } & M6-A & 0.003 & 0.068 & 0.930 & -10.6 & -11.8 & -12.3 & -29.3 & 0.086 & 0.003 & 0.026 \\
\hline & M6-B & 0.609 & 0.324 & 0.067 & -2.6 & 13.9 & -6.2 & -9.4 & 0.025 & 0.027 & 0.181 \\
\hline \multirow[t]{2}{*}{ Pyracylene } & M7-A & 0.000 & 0.329 & 0.671 & -4.9 & 6.9 & -7.2 & -13.3 & 0.067 & 0.014 & 0.128 \\
\hline & M7-B & 0.406 & 0.923 & -0.328 & 10.1 & 56.4 & 4.8 & 24.2 & $\mathrm{a}$ & 0.050 & 0.676 \\
\hline \multirow[t]{2}{*}{ Phenanthrene } & M8-A & 0.007 & 0.091 & 0.902 & -11.4 & -14.7 & -13.0 & -31.5 & 0.082 & 0.005 & 0.045 \\
\hline & M8-B & 0.189 & 0.409 & 0.402 & -6.8 & -0.1 & -9.4 & -20.0 & 0.053 & 0.025 & 0.255 \\
\hline \multirow[t]{2}{*}{ Acenaphthylene } & M9-A & 0.011 & 0.192 & 0.797 & -9.6 & -8.7 & -11.4 & -26.4 & 0.070 & 0.013 & 0.114 \\
\hline & M9-B & 0.337 & 0.703 & -0.039 & 2.2 & 33.7 & -1.8 & 4.6 & $\mathrm{a}$ & 0.045 & 0.578 \\
\hline \multirow[t]{2}{*}{ Biphenylene } & M10-A & 0.000 & 0.193 & 0.807 & -6.7 & -0.7 & -8.1 & -17.3 & 0.088 & 0.008 & 0.066 \\
\hline & M10-B & 1.360 & 0.571 & -0.930 & 17.4 & 87.0 & 7.5 & 35.7 & $\mathrm{a}$ & 0.048 & 0.297 \\
\hline \multirow[t]{2}{*}{ Banzocyclobutadiene } & M11-A & 0.001 & 0.501 & 0.497 & -4.0 & 8.4 & -5.4 & -9.5 & 0.080 & 0.022 & 0.191 \\
\hline & M11-B & 0.910 & 1.526 & -1.437 & 20.2 & 96.2 & 10.7 & 44.1 & $\mathrm{a}$ & 0.071 & 1.047 \\
\hline Pyridine & M12 & -0.006 & 0.001 & 1.005 & -9.5 & -15.2 & -12.5 & -31.6 & 0.097 & 0.001 & 0.001 \\
\hline Pyrimidine & M13 & 0.015 & 0.000 & 0.985 & -7.5 & -11.8 & -11.7 & -29.8 & 0.089 & 0.005 & 0.003 \\
\hline Triazine & M14 & 0.023 & 0.000 & 0.977 & -5.3 & -6.9 & -10.8 & -27.0 & 0.075 & 0.013 & 0.000 \\
\hline \multirow[t]{2}{*}{ Quinoline } & M15-A & 0.018 & 0.190 & 0.792 & -11.0 & -14.7 & -12.6 & -30.7 & 0.072 & 0.015 & 0.125 \\
\hline & M15-B & 0.008 & 0.161 & 0.830 & -9.1 & -11.5 & -12.1 & -29.5 & 0.071 & 0.017 & 0.121 \\
\hline Cyclohexane & M16 & 5.340 & 0.000 & -4.340 & -2.1 & 23.5 & -2.0 & 3.1 & 0.007 & 0.091 & $\mathrm{~b}$ \\
\hline Cyclohexane & M17 & 2.955 & 1.647 & -3.601 & -1.6 & 18.1 & -3.6 & -1.8 & 0.019 & 0.089 & $\mathrm{~b}$ \\
\hline Cyclohexa-1,4-diene & M18 & 0.779 & 1.984 & -1.763 & 1.5 & 25.4 & -0.8 & 2.9 & 0.014 & 0.084 & $\mathrm{~b}$ \\
\hline Cyclohexa-1,3-diene & M19 & 0.931 & 2.207 & -2.138 & 3.2 & 30.0 & 0.8 & 7.3 & 0.031 & 0.078 & $\mathrm{~b}$ \\
\hline
\end{tabular}

${ }^{\text {aPDI }}$ cannot be computed for non-6-MRs.

${ }^{\mathrm{b} N o n p l a n a r}$ molecules that prevent easy and exact $\sigma-\pi$ separation.

The second term of the right-hand side of Eq. (14) is what Krygowski and Cyrański call dearomatization. It has two contributions: the first contribution (the EN term) arises from the difference between the average distance in the ring and that calculated from the minimization of the deformation energy due to the extension of the single bonds and the compression of the double ones; the second contribution (the GEO term) is the bond-lengths variance of the bonds forming the ring. Equation (14) cannot be used directly as it is for the heterocyclic systems since the averaging procedure is meaningless for heterogenic data. Thus, for heterocyclic species, we have followed the procedure described in Ref. 41. As can be seen in Fig. 3, the GEO component is the term which better correlates with FLU. This is not unexpected

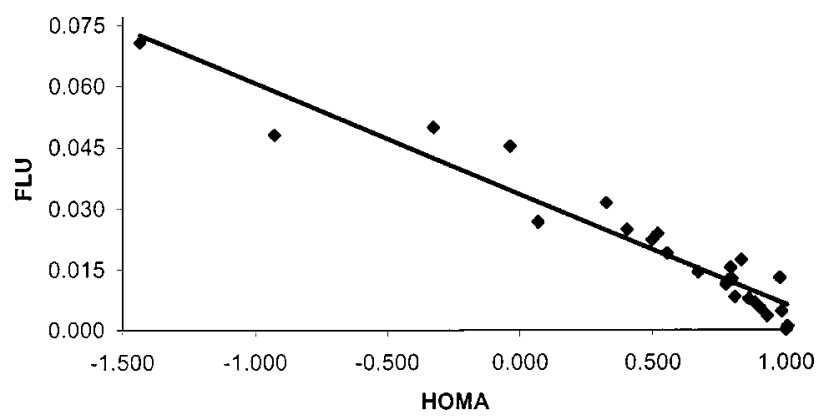

FIG. 2. Plot of FLU vs HOMA for the series of planar aromatic hydrocarbons M1-M15 (correlation coefficient $r=-0.957$ ). because in both the FLU and GEO expressions the most relevant component is a variance contribution. We also find a moderate correlation between the EN component of HOMA and FLU $(r=-0.789)$. The lower correlation of the EN term with FLU is in line with the fact that the main differences between FLU and HOMA found in the evaluation of the local aromatic character of ring $\mathbf{B}$ in triphenylene (M6) and quinoline (M15) arises from the EN term, which is particularly large for M6-B and surprisingly low for M15-B. On the other hand, for triazine (M14), both the EN and GEO terms are responsible for the HOMA overestimation (as compared to the rest of analyzed indices) of the local aromatic

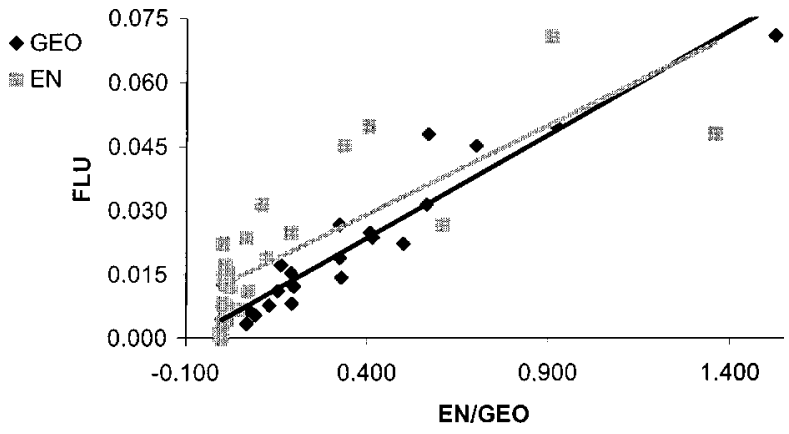

FIG. 3. Plot of FLU vs GEO $(r=0.952)$ and EN $(r=0.788)$ with FLU for the series of planar aromatic hydrocarbons M1-M15. 


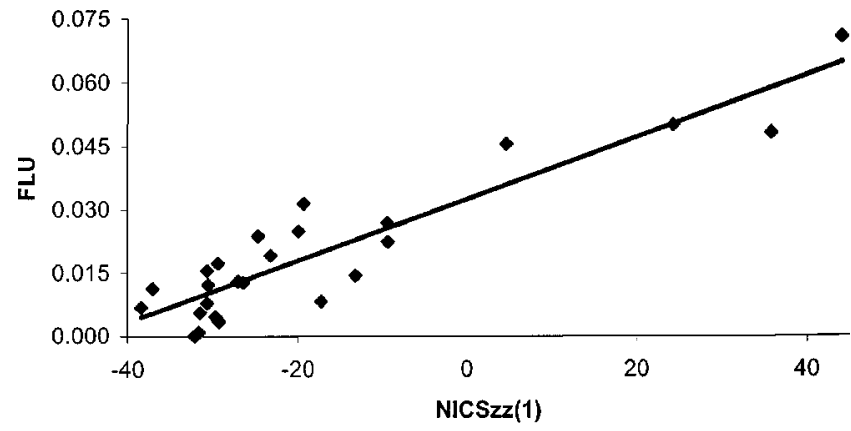

FIG. 4. Plot of $\operatorname{NICS}_{z z}(1)$ vs FLU values for the molecules M1-M15 ( $r$ $=0.918$ ).

character of M14. We note in passing that for systems having HOMA values larger than 1 , it is possible to have negative values for the EN term. ${ }^{4,41,42}$

\section{NICS results}

Table I collects the results of $\operatorname{NICS}(0), \operatorname{NICS}_{z z}(0)$, $\operatorname{NICS}(1)$, and $\operatorname{NICS}_{z z}(1)$. The differences between $\operatorname{NICS}(0)$ and $\mathrm{NICS}_{z z}(0)$ give a quantitative idea of the spurious information arising from the electron flow perpendicular to the molecular plane. ${ }^{43}$ The rings showing a larger departure from the linear regression of $\operatorname{NICS}(0)$ versus $\operatorname{NICS}_{z z}(0)$ are those corresponding to compounds having heteroatoms and/or fused rings. Interestingly, these deviations almost disappear when plotting NICS(1) against $\operatorname{NICS}_{z z}(1)$. These results suggest that ring currents in adjacent rings and/or the nitrogen in-plane electron cloud may mask the out-of-planeinduced magnetic field and that these effects are less notorious when considering only out-of-plane $\mathrm{NICS}_{z z}$ values. In general, there is a good linear relationship between the four different NICS values given in Table I and the FLU index $(r=0.90-0.92)$. Relevant differences between the various plots of FLU versus NICS are not observed, although the $\mathrm{NICS}_{z z}(1)$ values yield the best-fitted line (Fig. 4). It is worth noting that the local aromaticity of the inner rings of acenes such as rings $\mathbf{B}$ in anthracene and naphthacene are especially overestimated by all NICS measures as compared to the rest of aromaticity descriptors analyzed. Indeed, absolute NICS values are larger for the inner rings of anthracene and naphthacene than for benzene itself, whereas benzene is the most aromatic 6-MR of the series according to the FLU, HOMA, and PDI indices. These results give support to the claimed overestimation by NICS of the local aromaticity of the central rings in polyacenes. ${ }^{9,44}$

\section{PDI results}

The last index to be analyzed here is the PDI descriptor of local aromaticity. ${ }^{23}$ Due to its definition, this index can only be applied to analyze the local aromaticity of 6-MRs. Figure 5 displays the correlation between PDI and FLU for all 6-MRs appearing along the M1-M15 series. In general, there is a moderately good agreement $(r=-0.840)$ on the orderings yielded by these two criteria, the main exception being the 6-MR of benzocyclobutadiene, to which PDI attributes a high aromatic character, while HOMA (0.497) and

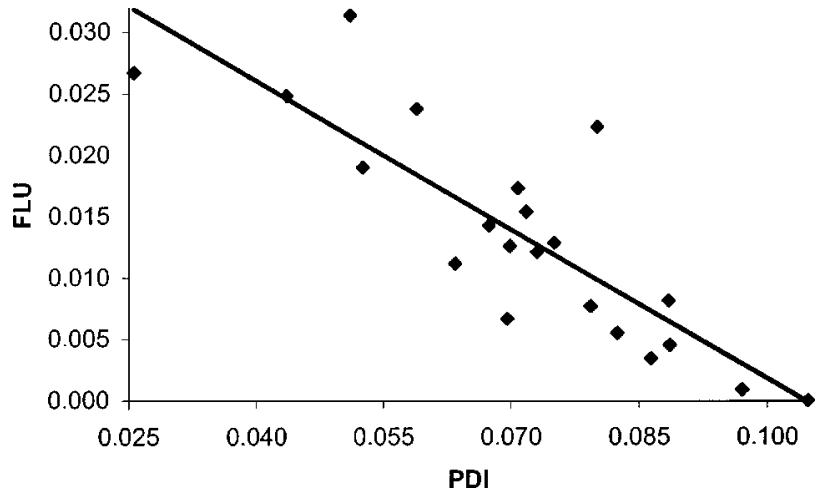

FIG. 5. Plot of PDI vs FLU values for the six-membered rings of molecules M1-M7-A, M8, M9-A, M10-A, M11-A, M12-M15 $(r=-0.840)$.

NICS(0) [not only NICS(0) but also $\operatorname{NICS}_{z z}(0), \operatorname{NICS}(1)$, and $\operatorname{NICS}_{z z}(1)$ values show that the 6-MR of benzocyclobutadiene has a low or moderate aromatic character] $(-4.0)$ indicators attributed it to a low or moderate aromaticity. Despite being reasonable, the correlation between FLU and PDI is lower than that shown by FLU with HOMA or NICS. This is surprising if one considers that the FLU and PDI definitions are based on the same manifestation of aromaticity, namely, the electronic delocalization in aromatic molecules.

\section{B. The aromaticity of the M16-M19 systems}

In this section, the ordering of aromatic character in the M16-M19 and M1 series given by the different indices is analyzed. M16 and M17 are nonplanar species, while M18, M19, and M1 systems possess a completely planar molecular structure. Due to the increase in unsaturated character along this series, an intensification of aromaticity is expected from M16 to M19 and especially to M1. Indeed, all indices show the predictable increase of aromaticity from planar M19 to M1. However, the only indices that more or less reproduce the anticipated trend along the full series are HOMA and FLU. Nonetheless, these two indices disagree on deciding the aromaticity of cyclohexa-1,4-diene (M18) and cyclohexa-1,3-diene (M19). Although both indices assign a similar low aromaticity for these compounds, HOMA gives a greater aromaticity to M18, while FLU asserts that M19 has a higher aromatic character. Thus, in contrast to HOMA, the FLU index succeeds in assigning a larger aromatic character to M19, which has two consecutive $\pi$-electron bonds, as opposed to M18, which has the $\pi$-electron systems disconnected.

On the other hand, when splitting the HOMA index on its GEO and EN contributions, we can see the expected trend with increasing aromaticity (see Fig. 6), i.e., stemming energy (EN term) when getting closer to the unsaturated delocalized picture of benzene, while symmetry (GEO term) on the structure achieves its minima on benzene and cyclohexane, and intermediate values for the nonsymmetric molecules M17-M19.

For M16-M19, NICS(0) provides a trend completely opposed to the expected one. The out-of-plane component of the NICS values gives somewhat better results. This notwithstanding, $\operatorname{NICS}_{z z}(0)$ and $\operatorname{NICS}_{z z}(1)$ values fail in predicting 


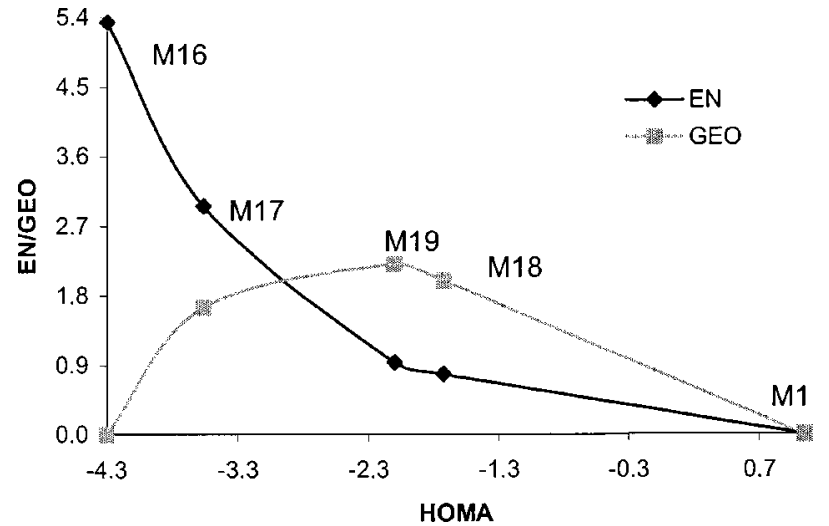

FIG. 6. Plot of GEO and EN vs HOMA values for the compounds M16M19 and M1.

cyclohexene (M17) as more aromatic than cyclohexadienes, and cyclohexa-1,3-diene as less aromatic than cyclohexane (M16). Moreover, the out-of-plane component is not easily related to the $\pi$ electrons for nonplanar molecules. In a previous work, ${ }^{37}$ Schleyer et al. showed that the $\mathrm{NICS}_{\pi}$ (NICS values obtained considering only the contribution of $\pi$ orbitals) reproduce the expected trend in aromaticity for the M18, M19, and M1 species. Since this $\mathrm{NICS}_{\pi}$ cannot be applied to nonplanar molecules, M16 and M17 were not analyzed using this index. ${ }^{37}$ Finally, PDI values fail when predicting a lower aromatic character for cyclohexa-1,4-diene (M18) as compared to cyclohexene. Apparently, the aromaticity in cyclohexene is somewhat overestimated by PDI because the nonplanar nature of this species makes the basins of carbons on para-position spatially closer than in 1,4-cyclohexadiene. This causes an increase of DIs in cyclohexene as compared to cyclohexa-1,4-diene.

\section{The $\mathrm{FLU}_{\pi}$ index of aromaticity}

The main shortcoming of the FLU index, as it is for the HOMA descriptor of aromaticity, is the need for reference parameters. At variance with PDI, NICS, and other indices, FLU and HOMA require archetypical aromatic molecules containing specific bonds as a reference. The original definition of the FLU index, based on the DIs that account for the sharing between a certain pair of atoms, enables the possibility of splitting this index in terms of orbital contributions. Gathering the $\pi$ contribution treated the way reported in Sec. II opens the possibility of calculating a FLU index free of reference parameters. Named hereafter as aromatic $\pi$ fluctuation index $\left(\mathrm{FLU}_{\pi}\right)$, this aromaticity criterion is calculated as follows:

$\mathrm{FLU}_{\pi}=\frac{1}{n} \sum_{A-B}^{\text {ring }}\left\{\left[\frac{\mathrm{Flu}_{\pi}(A \rightarrow B)}{\mathrm{Flu}_{\pi}(B \rightarrow A)}\right]^{\delta} \frac{\delta_{\pi}(A, B)-\delta_{a v}(A, B)}{\delta_{a v}(A, B)}\right\}^{2}$,

where $\delta_{a v}$ is the average value of the $\delta_{\pi}$, that is, the $\pi$ component of the DI, and $\mathrm{Flu}_{x}(A \rightarrow B)$ is calculated as in Eq. (9) using also only the $\pi$ component of the DIs. Correlation of $\mathrm{FLU}_{\pi}$ with FLU is excellent ( $r=0.973$, see Fig. 7 ), thus proving the similarity between FLU and $\mathrm{FLU}_{\pi}$ approaches. Correlations of $\mathrm{FLU}_{\pi}$ with HOMA, NICS, and PDI are also

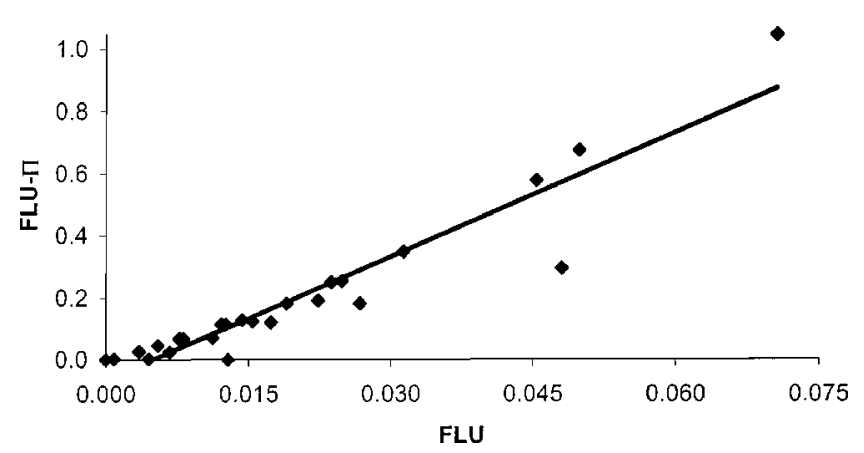

FIG. 7. FLU ${ }_{\pi}$ vs FLU representation for the series of planar aromatic hydrocarbons M1-M15 $(r=0.973)$.

found to be reasonable. An advantage of $\mathrm{FLU}_{\pi}$ with respect to FLU, apart from the fact that no reference parameters are required, is that the former gives values spread in wider ranges. For these reasons, we suggest the use of the $\mathrm{FLU}_{\pi}$ index for evaluation of aromaticity in planar species since no reference systems are necessary, while the FLU index is preferred when analyzing nonplanar systems, for which an exact $\sigma-\pi$ separation is not possible. Finally, we recommend using these FLU indices in conjunction with other differently based descriptors of aromaticity (HOMA, NICS, PDI,...) to account for the multidimensional character of aromaticity. $4,6,26,27$

\section{CONCLUSIONS}

While aromaticity has always been associated with the cyclic circulation of $\pi$ electrons, few attempts have been done until now to quantify this phenomenon by analyzing electron delocalization. To this end, we have defined in this work the aromatic fluctuation index (FLU), which not only analyzes the amount of electron sharing between contiguous atoms, which should be substantial in aromatic molecules, but also takes into account the similarity of electron sharing between adjacent atoms. We have demonstrated that the FLU index and its $\pi$ analog, the $\mathrm{FLU}_{\pi}$ descriptor, are simple and efficient probes for aromaticity. We have shown that, for a series of planar PAHs, FLU and $\mathrm{FLU}_{\pi}$ correlate well, with few exceptions, with other already existing independent local aromaticity parameters, such as the HOMA descriptor based on structural criteria, the NICS parameter founded on magnetic properties, and the PDI index derived from the analysis of electron delocalization, which are of common use nowadays. It remains to be seen whether these indices can be applied to discuss the aromaticity of novel inorganic compounds recently synthesized. ${ }^{45}$ Research in this direction is currently under way in our laboratory.

Note added in proof. It is worth noting that, recently, a new HOMA-like aromaticity index $\theta$, which bears some resemblance to the present FLU index, has been defined by replacing the bond distances in Eq. (1) by the corresponding delocalization indices [C. F. Matta and J. Hernández-Trujillo, J. Phys. Chem. A 107, 7496 (2003)]. 


\section{ACKNOWLEDGMENTS}

Financial help has been furnished by the Spanish MCyT Project Nos. BQU2002-0412-C02-02 and BQU2002-03334 and by the DURSI Project No. 2001SGR-00290. One of the authors (M.S.) is indebted to the DURSI of the Generalitat de Catalunya for financial support through the Distinguished University Research Promotion, 2001. Another author (E.M.) thanks the Secretaría de Estado de Educación y Universidades of the MECD for the Doctoral Fellowship No. AP2002-0581. The authors also thank the Center de Supercomputació de Catalunya (CESCA) for partial funding of computer time. See Ref. 46 for supporting information: Tables SI-1 with HF/6-31G(d) NICS at the RCP and SI-2 with HOMA, NICS, FLU, and PDI values for selected systems at the HF/6-311+ $+G(d, p)$ level of calculation.

${ }^{1}$ P. V. R. Schleyer, Chem. Rev. (Washington, D.C.) 101, 1115 (2001).

${ }^{2}$ F. Sondheimer, Pure Appl. Chem. 7, 363 (1964).

${ }^{3}$ P. V. R. Schleyer and H. Jiao, Pure Appl. Chem. 68, 209 (1996).

${ }^{4}$ T. M. Krygowski and M. K. Cyrański, Chem. Rev. (Washington, D.C.) 101, 1385 (2001).

${ }^{5}$ F. De Proft and P. Geerlings, Chem. Rev. (Washington, D.C.) 101, 1451 (2001).

${ }^{6}$ A. R. Katritzky, K. Jug, and D. C. Oniciu, Chem. Rev. (Washington, D.C.) 101, 1421 (2001).

${ }^{7}$ J. Gomes and R. B. Mallion, Chem. Rev. (Washington, D.C.) 101, 1349 (2001); M. N. Glukhovtsev, J. Chem. Educ. 74, 132 (1997).

${ }^{8}$ S. W. Slayden and J. F. Liebman, Chem. Rev. (Washington, D.C.) 101, 1541 (2001).

${ }^{9}$ T. M. Krygowski, M. K. Cyrański, Z. Czarnocki, G. Häfelinger, and A. R. Katritzky, Tetrahedron 56, 1783 (2000).

${ }^{10}$ J. Kruszewski and T. M. Krygowski, Tetrahedron Lett. 13, 3839 (1972).

${ }^{11}$ T. M. Krygowski, J. Chem. Inf. Comput. Sci. 33, 70 (1993).

${ }^{12}$ P. V. R. Schleyer, C. Maerker, A. Dransfeld, H. Jiao, and N. J. R. van Eikema Hommes, J. Am. Chem. Soc. 118, 6317 (1996).

${ }^{13}$ V. I. Minkin, M. N. Glukhovtsev, and B. Y. Simkin, Aromaticity and Antiaromaticity: Electronic and Structural Aspects (Wiley, New York, 1994).

${ }^{14}$ P. George, M. Trachtman, C. W. Bock, and A. M. Brett, J. Chem. Soc., Perkin Trans. 2 1977, 1036 ; M. N. Glukhovtsev and P. V. R. Schleyer, Chem. Phys. Lett. 198, 547 (1992); C. H. Suresh and N. Koga, J. Org. Chem. 67, 1965 (2002).

${ }^{15}$ K. Jug, J. Org. Chem. 48, 1344 (1983).

${ }^{16}$ S. T. Howard and T. M. Krygowski, Can. J. Chem. 75, 1174 (1997).

${ }^{17}$ G. P. Bean, J. Org. Chem. 63, 2497 (1998); N. Sadlej-Sosnowska, ibid. 66, 8737 (2001).

${ }^{18}$ A. Savin, R. Nesper, S. Wengert, and T. F. Fassler, Angew. Chem., Int. Ed. Engl. 36, 1809 (1997).

${ }^{19}$ A. D. Becke and K. E. Edgecombe, J. Chem. Phys. 92, 5397 (1990).

${ }^{20}$ D. B. Chesnut and L. J. Bartolotti, Chem. Phys. 253, 1 (2000); J. C. Santos, W. Tiznado, R. Contreras, and P. Fuentealba, J. Chem. Phys. 120, 1670 (2004); C. Lepetit, M. B. Nielsen, F. Diederich, and R. Chauvin, Chem.-Eur. J. 9, 5056 (2003)

${ }^{21}$ R. F. W. Bader and M. E. Stephens, J. Am. Chem. Soc. 97, 7391 (1975).

${ }^{22}$ X. Fradera, M. A. Austen, and R. F. W. Bader, J. Phys. Chem. A 103, 304 (1999).

${ }^{23}$ J. Poater, X. Fradera, M. Duran, and M. Solà, Chem.-Eur. J. 9, 400 (2003).

${ }^{24}$ R. F. W. Bader, Acc. Chem. Res. 18, 9 (1985); R. F. W. Bader, Atoms in
Molecules: A Quantum Theory (Clarendon, Oxford, 1990); R. F. W. Bader, Chem. Rev. (Washington, D.C.) 91, 893 (1991).

${ }^{25}$ J. Poater, X. Fradera, M. Duran, and M. Solà, Chem.-Eur. J. 9, 1113 (2003)

${ }^{26}$ M. K. Cyrański, T. M. Krygowski, A. R. Katritzky, and P. V. R. Schleyer, J. Org. Chem. 67, 1333 (2002).

${ }^{27}$ A. R. Katritzky, M. Karelson, S. Sild, T. M. Krygowski, and K. Jug, J. Org. Chem. 63, 5228 (1998).

${ }^{28}$ A. Savin, A. D. Becke, J. Flad, R. Nesper, H. Preuss, and H. G. Vonschnering, Angew. Chem., Int. Ed. Engl. 30, 409 (1991); B. Silvi and A. Savin, Nature (London) 371, 683 (1994)

${ }^{29}$ X. Fradera, J. Poater, S. Simon, M. Duran, and M. Solà, Theor. Chem. Acc. 108, 214 (2002).

${ }^{30}$ R. F. W. Bader, in Localization and Delocalization in Quantum Chemistry, edited by O. Chalvet, R. Daudel, S. Diner, and J. P. Malrieu (Reidel, Dordrecht, 1975), Vol. I, p. 15; P. Claverie and S. Diner, ibid., edited by O. Chalvet, R. Daudel, S. Diner, and J. P. Malrieu (Reidel, Dordrecht, 1976), Vol. II, p. 395

${ }^{31}$ A. Savin, B. Silvi, and F. Colonna, Can. J. Chem. 74, 1088 (1996); S. Noury, A. Colonna, and A. Savin, J. Mol. Struct.: THEOCHEM 450, 59 (1998).

${ }^{32}$ W. J. Hehre, R. Ditchfield, and J. A. Pople, J. Chem. Phys. 56, 2257 (1972); P. C. Hariharan and J. A. Pople, Theor. Chim. Acta 28, 213 (1973); M. Francl, W. J. Pietro, W. J. Hehre, J. S. Binkley, M. S. Gordon, D. J. Frees, and J. A. Pople, J. Chem. Phys. 77, 3654 (1982).

${ }^{33}$ M. J. Frisch, G. W. Trucks, H. B. Schlegel et al., A11 GAUSSIAN 98, Revision A11, Pittsburgh, PA, 1998.

${ }^{34}$ W. J. Hehre, L. Radom, P. V. R. Schleyer, and J. A. Pople, Ab Initio Molecular Orbital Theory (Wiley, New York, 1986).

${ }^{35}$ K. Wolinski, J. F. Hilton, and P. Pulay, J. Am. Chem. Soc. 112, 821 (1990).

${ }^{36}$ I. Morao, B. Lecea, and F. P. Cossio, J. Org. Chem. 62, 7033 (1997); F. P. Cossio, I. Morao, H. J. Jiao, and P. V. R. Schleyer, J. Am. Chem. Soc. 121, 6737 (1999)

${ }^{37}$ P. V. R. Schleyer, M. Manoharan, Z. X. Wang, B. Kiran, H. J. Jiao, R. Puchta, and N. J. R. van Eikema Hommes, Org. Lett. 3, 2465 (2001).

${ }^{38}$ E. Matito, J. Poater, M. Duran, and M. Solà, J. Mol. Struct.: THEOCHEM (submitted).

${ }^{39}$ C. Corminboeuf, T. Heine, G. Seifert, P. V. R. Schleyer, and J. Weber, Phys. Chem. Chem. Phys. 6, 273 (2004).

${ }^{40}$ F. W. Biegler-König, R. F. W. Bader, and T.-H. Tang, J. Comput. Chem. 3, 317 (1982).

${ }^{41}$ T. M. Krygowski and M. C. Cyrański, Tetrahedron 52, 1713 (1996).

${ }^{42}$ T. M. Krygowski and M. C. Cyrański, Tetrahedron 52, 10255 (1996).

${ }^{43}$ P. Lazzeretti, in Progress in Nuclear Magnetic Resonance Spectroscopy, edited by J. W. Emsley, J. Feeney, and L. H. Sutcliffe (Elsevier, Amsterdam, 2000), Vol. 36, p. 1; P. Lazzeretti, Phys. Chem. Chem. Phys. 6, 217 (2004)

${ }^{44}$ M. V. Zhigalko, O. V. Shishkin, L. Gorb, and J. Leszczynski, J. Mol. Struct.: THEOCHEM 693, 153 (2004).

${ }^{45}$ X. Li, A. E. Kuznetsov, H.-F. Zhang, A. Boldyrev, and L.-S. Wang, Science 291, 859 (2001); F. De Proft, P. W. Fowler, R. W. A. Havenith, P. V. R. Schleyer, G. Van Lier, and P. Geerlings, Chem.-Eur. J. 10, 940 (2004); M. Lein, J. Frunzke, and G. Frenking, Angew. Chem., Int. Ed. 42, 1303 (2003); R. W. A. Havenith, P. W. Fowler, E. Steiner, S. Shetty, D. Kanhere, and S. Pal, Phys. Chem. Chem. Phys. 6, 285 (2004).

${ }^{46}$ See EPAPS Document No. E-JCPSA6-121-310448 for the NICS at the RCP and the HOMA, NICS, FLU, and PDI calculated at the HF/6-311 $++G(d, p)$ level of theory for selected systems. A direct link to this document may be found in the online article's HTML reference section. The document may also be reached via the EPAPS homepage (http:// www.aip.org/pubservs/epaps.html) or from ftp.aip.org in the directory /epaps/. See the EPAPS homepage for more information. 